\title{
Gmbak1 and gmfls2, two genes response to bacterials disease of soybean
}

\author{
Jianan Zou ${ }^{1}$, Dawei Xin ${ }^{1}$, and Qingshan Chen ${ }^{1, *}$ \\ ${ }^{1}$ Northeast Agricultural University, Harbin China.
}

\begin{abstract}
Soybean bacterial disease is a worldwide disease that can cause yield and quality decline. Although the recognition of soybean bacterial diseases has been reported in detail, but for the identification of resistant and susceptible cultivars, different researchers have different methods and standards. To detect the pathogenic effect of bacterial diseases, we first separated bacterial strains from soybean field. Finally, eight strains of soybean bacterial diseases were isolated and identified. By the screening the sequence of the bacterial 16S rDNA, seven of eight strains were identified belong to Pseudomonas, another strain belong to Xanthomonas. In order to study the relevant pathogen of soybean defense response, we used the isolated pathogen strain to inoculate the resistant cultivar Hefeng35 and sensitive cultivar Suinong14. Then the genes might involve in regulate soybean resistant to bacterial disease were analyzed, including GmBAK1 and GmFLS2. FLS2 homolog gene were Gm05g25830, Gm08g08810; BAK1 homolog gene were Gm15g05730, Gm08g07930, Gm10g36280, Gm05g24790, Gm20g31320, Gm05g24770, Gm08g19270, and Gm02g08360. By the qRT-PCR analysis we detected the gene expression pattern response to pathogen, we found the expression of Gm05g24770, Gm05g24790, and Glyma05g25830 showed significant different between Suinong14 and Hefeng35. We illustrated the GmBAK1 and GmFLS2 transcription level against bacterial speck disease pathogen infection and revealed preliminary the major gene, providing a reference direction for further research.
\end{abstract}

\section{Introduction}

Soybean originated in China, and then spread around the world with the circulation of commodity trade. However, soybean disease is the main factor affecting soybean yield. Soybean mosaic virus [1-3], soybean gray spot, downy mildew [4], bacterial leaf spot [5-7], etc. Due to the different ecological environment, cultivation system and varieties in different planting areas, soybean diseases will have obvious regional distribution characteristics.

\footnotetext{
*Corresponding author: qshchen@126.com
} 
Bacterial spot is a common bacterial disease of soybean, which occurs in the main production area of soybean. In the United States, soybean yield reduction caused by bacterial diseases can reach $4-40 \%$ [8-11]. It can be divided into three kinds: soybean bacterial spot, Pseudomonas syringae pv. Glycine (PSG) [12-14], soybean bacterial spot, Xanthomonas campestris. The pathogen of soybean bacterial wildfire is Pseudomonas syringae pv. Tabaci [15]. The most common soybean bacterial diseases are these two. It is mainly harmful to bean leaves. In China, it is mainly distributed in the main soybean producing areas of northeast and Huanghuaihai area, and the regional diseases of soybean planting in the north are more serious than those in the South [16]. Foreign countries are mainly distributed in Japan, Korean Peninsula, Australia, the United States, New Zealand and other countries. It is one of the major diseases in soybean production areas in the world, which can infect soybean, lunar bean, bean and other legume plants.

In recent years, scientists have been devoted to study how pathogens conquer the innate immunity of crops, develop new disease control methods, study the molecular mechanism and mechanism of plant disease resistance, and study the isolation and identification of resistance genes and their functions at the molecular level.

There are several pattern recognition receptors (PRRS) [17-18], which can recognize some common and conserved molecular motif pathogen related molecular patterns (PMAP) on plant cell membrane, and trigger innate immune response [19]. In the process of plant disease resistance, scientists found that Xa21 was involved in the immune response of Xoo gene in rice [20]. In Arabidopsis, fls2 can recognize flg22 [22], elf18 [23, 24 ]it is a conserved EF Tu gene sequence in bacteria, Arabidopsis can recognize its N-terminal acetylated peptide, which contains 18 amino acids, so it becomes elf18, which is the activator inducer of plant defense response). At the same time, AtFLS2 [26-28], LeFLS2 $[29,30]$ and LeFLS2 were also found in rice and tomato. The receptor proteins in these plants play an important role in activating the physiological processes of plant resistance and defense [31,32]. At present, the function of recognizing receptor related genes on soybean membrane is not clear.

Flg22 induces fls2 and BAK1 to form ligands and activate downstream defense response signaling pathway $[33,34]$. With the verification of the function of these proteins, the mechanism of plant immune signal transduction is gradually clear, and the plant immune signal pathway is gradually formed. BAK1, as the hub of plant immune signaling pathway, its function is particularly important.

\section{Materials and methods}

\subsection{Collection, isolation and identification of pathogen of soybean bacterial spot disease}

The diseased leaves were collected and separated from soybean fields, including Yanjiagang farm (No. 1-3) of Heilongjiang Beidahuang group, experimental practice base (No. 4-6) of Northeast Agricultural University, and diseased leaves (No. 7-8) collected by Jiamusi Academy of Agricultural Sciences.

Inoculate the bacterial solution onto King's medium B solid culture medium, store in refrigerator at $4{ }^{\circ} \mathrm{C}$ for standby after culture, and reproduce once every 3 weeks or so. The genomic DNA of bacteria was extracted. In this experiment, for the collected strains, at $28{ }^{\circ} \mathrm{C}, 180 \mathrm{rpm}$, culture medium for 24 hours, take $1-4 \mathrm{ml}, \mathrm{OD}_{600}=0.8$. Genomic DNA was extracted according to the extraction kit of Bactria genomic DNA. 16S rDNA gene of bacteria was amplified by $27 \mathrm{~F}, 1492 \mathrm{R}$ primers. After gene purification, it was linked with 
the pEasy clone vector, sequenced and compared with M13F and T7 sequencing primers. The detected sequence was stored in GenBank nucleic acid sequence database, eztaxon-e (http://eztaxon-e.ezbiocloud.net/). The $16 \mathrm{~S}$ rDNA sequence data were analyzed for homology.

\subsection{Excavation of BAK1 genes in Soybean}

Take the opposite real leaves with similar growth potential, spray the bacterial solution into the leaves from the back of the leaves, avoiding the main veins, using the electric high-pressure spray gun, and take it as the standard to see the wet area of the leaves at the inoculation site, so as to avoid over inoculation and damage to the leaves.

\section{Results}

\subsection{The bacterial pathogens isolation in soybean field}

In the field, bacterial spot disease is characterized by serious local disease. A certain plot or concentrated in a certain variety is obviously susceptible to the disease (Figure1). Therefore, the leaves with obvious disease are taken as materials for the isolation of pathogenic bacteria. Through the sampling in three places, it was found that soybean bacterial disease was common in Heilongjiang Province. From the field survey, the degree of bacterial spot disease was different in different years. After the rainstorm, the field investigation and sampling were carried out, which is conducive to the spread of pathogens and the growth of pathogens in humid environment. It is not the whole field disease, mainly concentrated in some susceptible varieties, and the leaf disease is obvious in the growing season. However, soybean bacterial spot disease can occur everywhere. At the beginning of the infected leaves, the color of the diseased spots is light brown, and there are chlorotic halos around. The diseased spots are 2-3mm. Subsequently, the disease spots expanded. When the disease was serious, the disease spots were densely distributed and the susceptible leaves were fragile and dry.

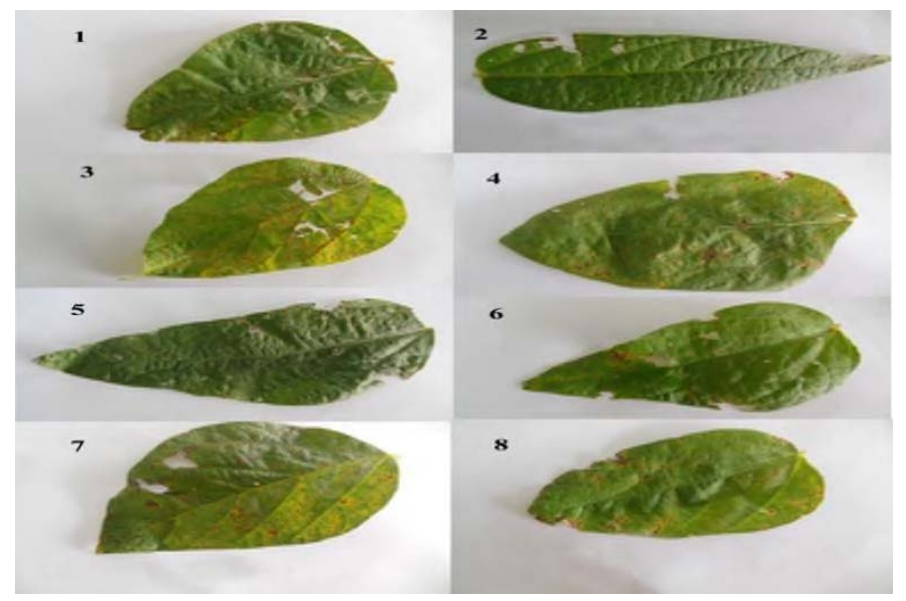

Fig. 1. Collection of diseased leaves. 
Note: sampling place: No. 1-3: Yanjiagang farm, Heilongjiang Province; No. 4-6: experimental practice base of Northeast Agricultural University; No. 7-8: Jiamusi Academy of Agricultural Sciences.

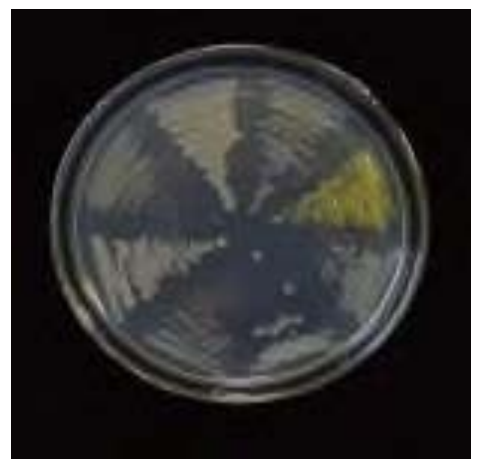

Fig. 2. Pathogen isolation on solid medium.

Note: clockwise No. 1-3: Yanjiagang farm, Heilongjiang Province, No. 4-6: experimental practice base of Northeast Agricultural University, No. 7-8: Jiamusi Academy of Agricultural Sciences.

\subsection{Identification of isolated strain}

The sequences of 16S rDNA was compared in eztaxon-e, and the experimental results are shown in Fig. 3. In this experiment, the strains Psgneau2, Psegneau4, Psegneau5, Psegneau6, Psegneau8 predicted that the closest strain was Pseudomonas ficurectae JCM 2400 (T). Psgneau1, Psegneau3, predicted that the closest strain was Pseudomonas congelans DSM 1439 (T). Xaneau7 predicted that the closest strain was Xanthomonas vasicola LMG 736(T). Therefore, Psgneau1-6, Psgneau8 were identified as Pseudomonas spp. The pathogen of xaneau7 is Xanthomonas.

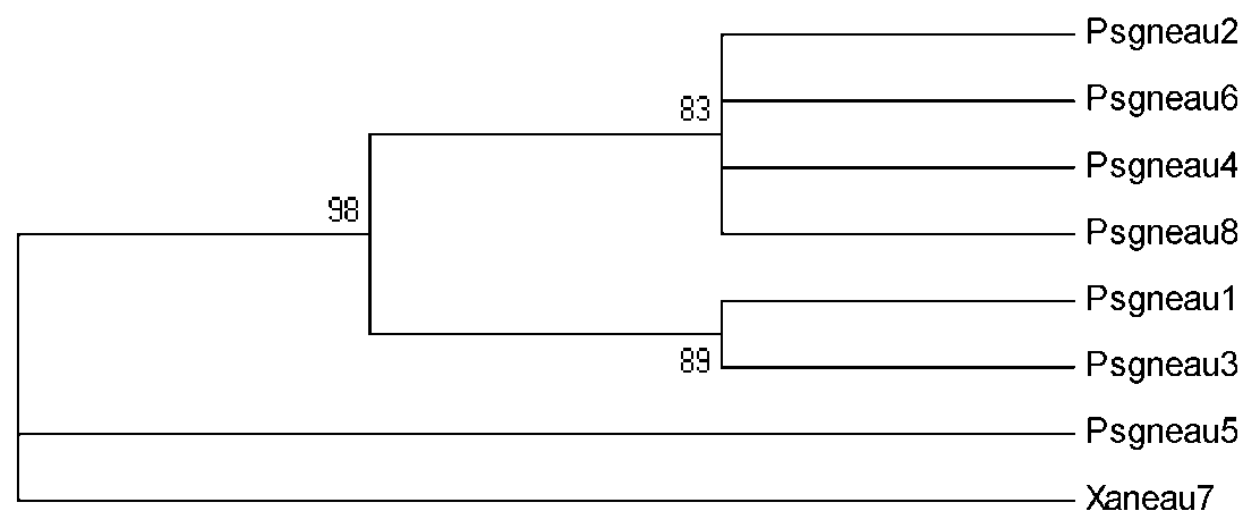

Fig. 3. Phylogenetic tree bacterial gene. 


\subsection{Gene expression analysis}

Four days after inoculation, compared with the control Suinong8, Suinong14 was susceptible at the level of 0.001 , Dongnong50 at the level of 0.05 , Hefeng35 at the level of 0.05 . There was no significant difference between other varieties and Suinong8. The results showed that the pathogenicity of the same bacterial spot of soybean to different soybean varieties was different. According to ten soybean varieties inoculated with strain 2, Hefeng35 and Suinong14 were selected to inoculate with psgneau2. Three genes, Glyma02g08360, Glyma20g31320 and Glyma08g07930, all showed an increase in relative gene expression in Hefeng35, while in suinong14, the relative gene expression remained unchanged. Among them, the expression level of Glyma02g08360 reached a certain peak $0.5 \mathrm{~h}$ after the exposure of Hefeng35, the expression amount was 1.67 times of the initial expression amount; the expression level of Glyma20g3320, Glyma08g07930 reached a certain peak 1 hour after the exposure of Hefeng35, and then decreased to the initial expression amount, the expression amount was divided into 5 times and 2 times of the initial relative expression amount (Figure 4 A-C).

Glyma08g19270 showed an increase in the relative expression of Hefeng35, which was then relatively stable. The relative expression of Suinong14 decreased at first, then recovered to the initial relative expression after $0.5 \mathrm{~h}$. At $0.5 \mathrm{~h}$ after inoculation, the expression level of Hefeng35 reached a certain peak, and the relative expression amount was 1 times of the initial relative expression amount; while the relative expression amount of Suinong14 decreased, and reached a stable level at $0.5 \mathrm{~h}$, and the initial relative expression amount was 2 times of the relative expression amount after 0.5h (Figure 4D). Glyma05g24770 showed an increase in the relative gene expression in Suinong14, while in Hefeng35, the relative gene expression was basically unchanged. One hour after Suinong 14 was inoculated, the expression level reached a certain peak, and the expression amount was twice of the initial expression amount (as shown in Figure 4E).

Glyma10g36280 showed an increase in expression in Hefeng35, which was later stable. The relative expression of suinong14 fluctuated with time. The relative expression of Hefeng35, a disease resistant variety, increased within $1 \mathrm{~h}$ after inoculation and reached the highest value within $3 \mathrm{~h}$. The relative expression was about 5 times of the initial relative expression and still had a high relative expression within 3-6h. The relative expression of Suinong14, a disease susceptible variety, also fluctuated within a small range. The relative expression of Hefeng 35 within $0.5 \mathrm{~h}$ and $3 \mathrm{H}$ was twice of the initial relative expression (as shown in Fig. 4F).

The relative expression of Glyma15g05730 increased in Hefeng35 and Suinong14, and reached the highest level in $3 \mathrm{H}$. The relative expression of Hefeng35 in $3 \mathrm{H}$ was 4 times of the initial level. In Suinong14, the relative expression of gene was temporarily reduced in $0.5 \mathrm{~h}$, and increased to the maximum in $3 \mathrm{~h}$, and the relative expression was 2.3 times of the initial level (as shown in Fig. 4G). The relative expression of Glyma05g24790 in Hefeng35 was almost the same, while that in suinong14 was lower. In Suinong14, the relative expression of $0.5 \mathrm{~h}$ is 0.25 times of the initial level, and then the relative expression of gene is at a relatively stable level (Fig. $4 \mathrm{H})$. 
A

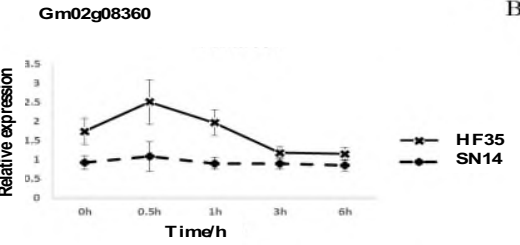

C

Gm08g07930

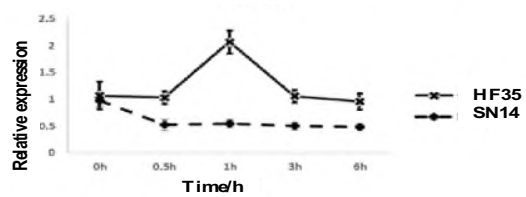

E

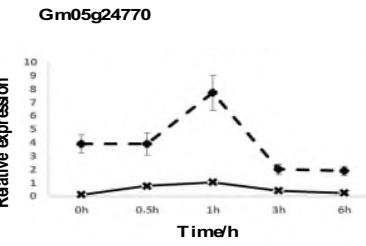

G

Gm05g05730

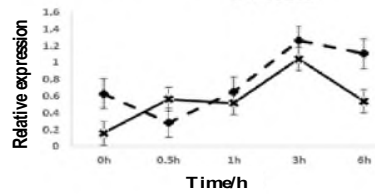

D

F
Gm20g31320

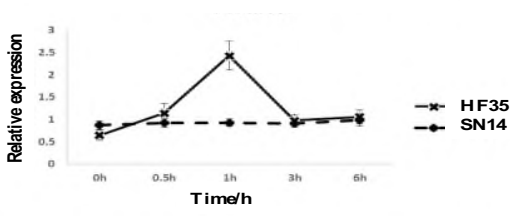

Gm08g19270

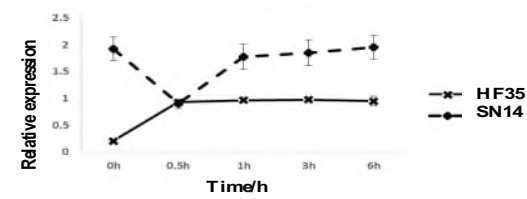

Gm10g36280
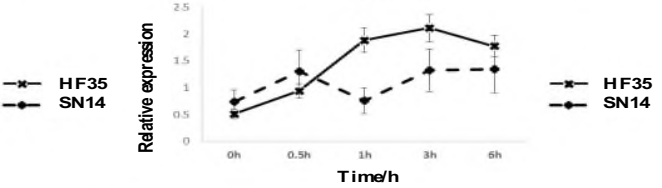

Gm05g24790

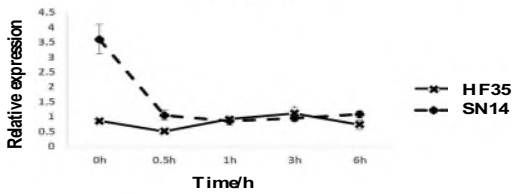

Fig. 4. Genes expression identification in Hefeng35 (HF35) and Suinong14 (SN14).

\section{Discussion}

At present, there are 8 BAK1 genes in soybean, but it is clear that there is one BAK1 gene in Arabidopsis. The amino acid sequence length of Arabidopsis is 663aa, and the amino acid sequence length of soybean BAK1 is 616-625aa, which is shorter than that of Arabidopsis. Contains similar domain sequences. The number of BAK1 related genes in soybean is more than that in Arabidopsis, which may be due to the fact that soybean is an ancient tetraploid with 20 pairs of chromosomes and its genome is relatively complex. Arabidopsis has only 5 pairs of chromosomes, the genome is small and relatively simple. In this experiment, the resistant variety Hefeng 35 and the susceptible variety Hefeng25 were inoculated respectively. Reference method [6], select five sampling time points to explore the specific expression of related genes at different times. In the resistant variety Hefeng35, BAK1 gene can be expressed in large quantity in the shortest time of $0.5 \mathrm{~h}$, and peak value in the latest time of 3 hours; in the susceptible variety Suinong14, the expression of BAK1 gene is not obvious in Hefeng35, and the expression quantity is basically unchanged or decreased. The response of BAK1 related genes to resistant varieties was significantly earlier than that of susceptible varieties. It was verified from the transcription level that the disease course of resistant varieties developed faster than that of susceptible varieties, and the signal transduction pathway of disease resistance needs to be studied. However, there are still two genes in the opposite situation, the expression of gm05g24770 increased in 
Suinong14, but remained unchanged in Hefeng35; the expression of gm05g24770 decreased in Suinong14, but remained unchanged in Hefeng35. At the transcription level, it was indicated that BAK1 was related to the resistance of the pathogen of bacterial spot disease of soybean, and the major genes that played the main role in the genes were preliminarily understood, but further research was needed.

\section{Conclusions}

We found that GmFLS2 and GmBAK1 coded genes, including Gm05g24770, Gm05g24790, and Glyma05g25830 are the candidate genes deserved to be further detected. Although many genes coded the proteins belong the GmFLS2 and GmBAK1 family, not all genes had the same response to bacterial pathogen between resistant and sensitive cultivars.

\section{Reference}

1. Y. Han, Z. Cheng, X. Zhao, et al. SSR marker assisted identification of resistance to soybean mosaic virus and Phytophthora root rot, Soybean science 32 (006): 740-743 (2013)

2. J. Liangyu. Preliminary study on the function of PR10 and PrP genes related to Phytophthora sojae root rot resistance (2013)

3. Li. B, Xue. J, sun D, et al. Research progress of Phytophthora root rot of Soybean Seed world 12 23-24 (2013)

4. Liu Shuxia, pan Dongmei, Wei Guojiang, et al. Toxic effect of four plant extracts on soybean cyst nematode, Heilongjiang Agricultural Science 11 46-49 (2013)

5. Zheng Yanan, Tian Feng, Chen Jingsheng, et al. Changes of cold resistant substances in soybean cyst nematode during dormancy, Journal of plant protection, 40 (3): 287-288 (2013)

6. Tao Caihong, Pan Yan, Du Xiao. Preliminary report on the control effect of different fungicides on soybean downy mildew in Huanxian County, Agricultural science and technology and information 3, 43-44 (2013)

7. Zhang Mingrong, Wu Haiying, he Zemin, et al. Occurrence and control of main diseases, pests and weeds of soybean interplanting in Sichuan Province Soybean science and technology, 4, 021 (2013)

8. J. A. Wrather, S. R. Koenning. Effects of diseases on soybean yields in the United States 1996 to 2007[J]. Plant Health Prog. 10 (2009)

9. E.W. Park Effects of bacterial blight on soybean yield Plant disease, 70. (1986)

10. J. E. Cross, B. W. Kennedy, J. W. Lambert, et al. Pathogenic races of the bacterial blight pathogen of soybeans, Pseudomonas Glycinea, Plant Disease Reporter, 50(8), 557-560 (1966)

11. L. K. Prom, J. R. Venette. Races of Pseudomonas syringae pv. Glycinea on commercial soybean in eastern North Dakota, Plant disease 81(5 ) 541-544 (1997)

12. Ding Junjie. Research survey of resistance identification of soybean bacterial spot disease [J]. Heilongjiang Agricultural Science, (1): 132-134 (2013)

13. K. Chiku, K. Tsunemi, M. Yamamoto, et al. Defects in D-Rhamnosyl Residue Biosynthetic Genes Affect Lipopolysaccharide Structure, Motility, and Cell-Surface 
Hydrophobicity in Pseudomonas syringae Pathovar Glycinea Race 4, Bioscience, biotechnology, and biochemistry, 77(3), 505-510 (2013)

14. J. N. Worley, A. B. Russell, A. G. Wexler, et al. Pseudomonas syringae pv. tomato DC3000 CmaL (PSPTO4723), a DUF1330 family member, is needed to produce L-allo-isoleucine, a precursor for the phytotoxin coronatine, Journal of bacteriology, 195(2), 287-296 (2013)

15. H. C. McCann, E. H. A. Rikkerink, F. Bertels, et al. Genomic analysis of the kiwifruit pathogen Pseudomonas syringae pv. actinidiae provides insight into the origins of an emergent plant disease, PLoS pathogens, 9(7), e1003503 (2013)

16. Zhao Haihong. The causes of soybean bacterial macular disease and its control methods Heilongjiang Agricultural Science, 11, 159-160 (2013)

17. H. Abdel-Haleem, E. D. Wood, H. R. Boerma, et al. Registration of G08PR-394 and 09PR-80 Soybean Germplasm Lines with Diverse Pedigrees Journal of Plant Registrations, 7(3), 347-352 (2013)

18. S. Lee, D. S. Yang, S. R. Uppalapati, et al. Suppression of plant defense responses by extracellular metabolites from Pseudomonas syringae pv. tabaci in Nicotiana benthamiana BMC plant biology, 13(1), 65 (2013)

19. K. J. Gupta, Y. Brotman, S. Segu, et al. The form of nitrogen nutrition affects resistance against Pseudomonas syringae pv. phaseolicola in tobacco, Journal of experimental botany 64(2), 553-568 (2013)

20. Wang Fang. Identification and comprehensive control of soybean bacterial diseases [J]. Soybean bulletin, 5:009 (2007)

21. P. Broz, D. M. Monack. Newly described pattern recognition receptors team up against intracellular pathogens, Nature Reviews Immunology, 13(8), 551-565 (2013)

22. X. W. Wang, J. X. Wang Pattern recognition receptors acting in innate immune system of shrimp against pathogen infections, Fish \& shellfish immunology, 34(4), 981-989 (2013)

23. Y. Sun, L. Li, A. P. Macho, et al. Structural basis for flg22-induced activation of the Arabidopsis FLS2-BAK1 immune complex Science 342(6158), 624-628.

24. W. Y. Song, G. L. Wang, L. L. Chen, et al. A receptor kinase-like protein encoded by the rice disease resistance gene, Xa21, Science, 270(5243), 1804-1806 (2012)

25. L. Gómez-Gómez, T. Boller. FLS2: An LRR receptor-like kinase involved in the perception of the bacterial elicitor flagellin in Arabidopsis, Molecular cell, 5(6), 1003-1011 (2000)

26. K. Sawinski, S. Mersmann, S. Robatzek, et al. Guarding the green: pathways to stomatal immunity Molecular Plant-Microbe Interactions, 26(6): 626-632 (2013)

27. M. Albert, A. K. Jehle, U. Fürst, et al. A two-hybrid-receptor assay demonstrates heteromer formation as switch-on for plant immune receptors Plant physiology, 163(4) 1504-1509 (2013)

28. B. Schulze, T. Mentzel, A. K. Jehle, et al. Rapid heteromerization and phosphorylation of ligand-activated plant transmembrane receptors and their associated kinase BAK1 Journal of Biological Chemistry, 285(13): 9444-9451 (2010)

29. D. R. Hann, A .Domínguez-Ferreras, V. Motyka, et al. The Pseudomonas type III effector HopQ1 activates cytokinin signaling and interferes with plant innate immunity New Phytologist, 201(2): 585-598 (2014) 
30. D. R. Hann, A. Domínguez-Ferreras, V Motyka, et al. The Pseudomonas type III effector HopQ1 activates cytokinin signaling and interferes with plant innate immunity New Phytologist, 201(2): 585-598 (2014)

31. A Akamatsu, Wong $\mathrm{H}$ L , Fujiwara $\mathrm{M}$, et al . An OsCEBiP/OsCERK1-OsRacGEF1-OsRac1 module is an essential early component of chitin-induced rice immunity, Cell host \& microbe, 13(4): 465-476 (2013)

32. X. Chen, S. Zuo, B. Schwessinger, et al. An XA21-Associated Kinase (OsSERK2) regulates immunity mediated by the XA21 and XA3 immune receptors, Molecular Plant, ssu003 (2014)

33. D. Chinchilla, C. Zipfel, S. Robatzek, et al. A flagellin-induced complex of the receptor FLS2 and BAK1 initiates plant defence Nature, 448(7152), 497-500 (2007)

34. V. Göhre, T. Spallek, H. Häweker, et al. Plant pattern-recognition receptor FLS2 is directed for degradation by the bacterial ubiquitin ligase AvrPtoB, Current Biology, 18(23), 1824-1832 (2018) 